\title{
Adapting Hands-on Laboratory's Materials and Embedded Systems from Local Use to Remote Experimenting through Internet
}

\author{
Yassine Larbaoui \\ Electrical engineering department \\ University Hassan 1 \\ Settat, Morocco
}

\begin{abstract}
This paper presents the work of adapting hands-on laboratory's materials of NI Elvis and Quanser from in-poste exploit to online access and remote exploit for online experimenting, after analyzing different aspects of adapting any hands-on laboratory's material of in-place experimenting to remote exploit. This paper presents the work of developing a software multiplexing technique, and other techniques, to multiplex between different software codes and programs, in order to control different types of experiments in electronic of energy while using and sharing the same physical components and materials nearly simultaneously. In addition, this paper presents the work of creating web client interfaces; to use those embedded systems of NI Elvis and Quanser and their deployed experiments through the internet while relying on an e-learning platform of our remote lab to support their remote access. The principal advantage of conducted adaptations is sharing the same hardware and software resources between different experiments at the same time, while exploiting them locally and through the internet by multiusers.
\end{abstract}

Keywords:- Circuits experimenting; e-learning; embedded systems; experiments switching; software switching; remote experiments.

\section{INTRODUCTION}

Laboratory's tests, exercises and experiments are essential parts and indispensable for education, scientific research fields and technical investigation [1]. Hands-on laboratories [2], [3] are the most common forms of laboratories as experimenting territories and environments. These laboratories are offering to students, searchers and other users, the opportunity to experiment on physical materials and different systems of software and hardware; related or dedicated to research or educational purposes.

Generally, Hands-on laboratories are known for requiring high costs of materials, in addition of requiring more space and more maintenance staff [4]. Considering, as an example, electrical engineering areas [5] and micronanoscience [6], it would be accurate to say that these costs and requirements increase even higher where the quality of education calls for a large variety of experiments, as it is the case in robotics, automation, mechatronic engineering, etc.

\author{
Ahmed Naddami, Ahmed Fahli \\ Electrical engineering department \\ University Hassan 1 \\ Settat, Morocco
}

High investments of buying, deploying, supervising and exploiting experimental materials, in addition of the maintenance and safety costs, pose important limitations and call for serious consideration of other alternatives or complementary resources, to manage and reduce these cumulated costs. As relevant solutions, relying on resource exploit and sharing via offsite facilities with the use of distance access and online use through the internet, or utilizing the simulated/emulated versions of physical materials to solve the already mentioned limitations.

The basic concept of remote access and exploit of distant equipment and materials of laboratories [7] arises to be built naturally from telemetry and telecommunication technologies, especially the well-established trend of using computers; to use and transfer the control data, the recorded data and collected measurements from local lab apparatus to distant users.

Basically, in an experimental environment of a remote lab, the deployed workbench of instruments, equipment and other materials are not physically in front of the student, because they are intended to be provided and exploited using distance access through internet [8]. Relying on this approach of distance service and online use, experiments are enabled to be delocalized and supported to be performed geographically anywhere, as long as there will be a reliable internet connection.

Remote Laboratories have been focusing on specific solutions to respond to their requirements while solving specific technical and pedagogical problems. We can find a wide range of remote laboratories in literature with different characteristics and factors [9], assisting and supporting different educational fields and research subjects (electricity, electronics, robotics, optic, fluid mechanic, etc.), whereas their majority is commonly bound to restricted and defined sets of requirements. Those facts are explaining the recent paid attentions on working on the open architecture aspect of scalable and maintainable topologies of hardware and software [7], to address wide sets of experiments, which will help to make these laboratories extendedly adaptable to integrate and support new materials and various experiments. 


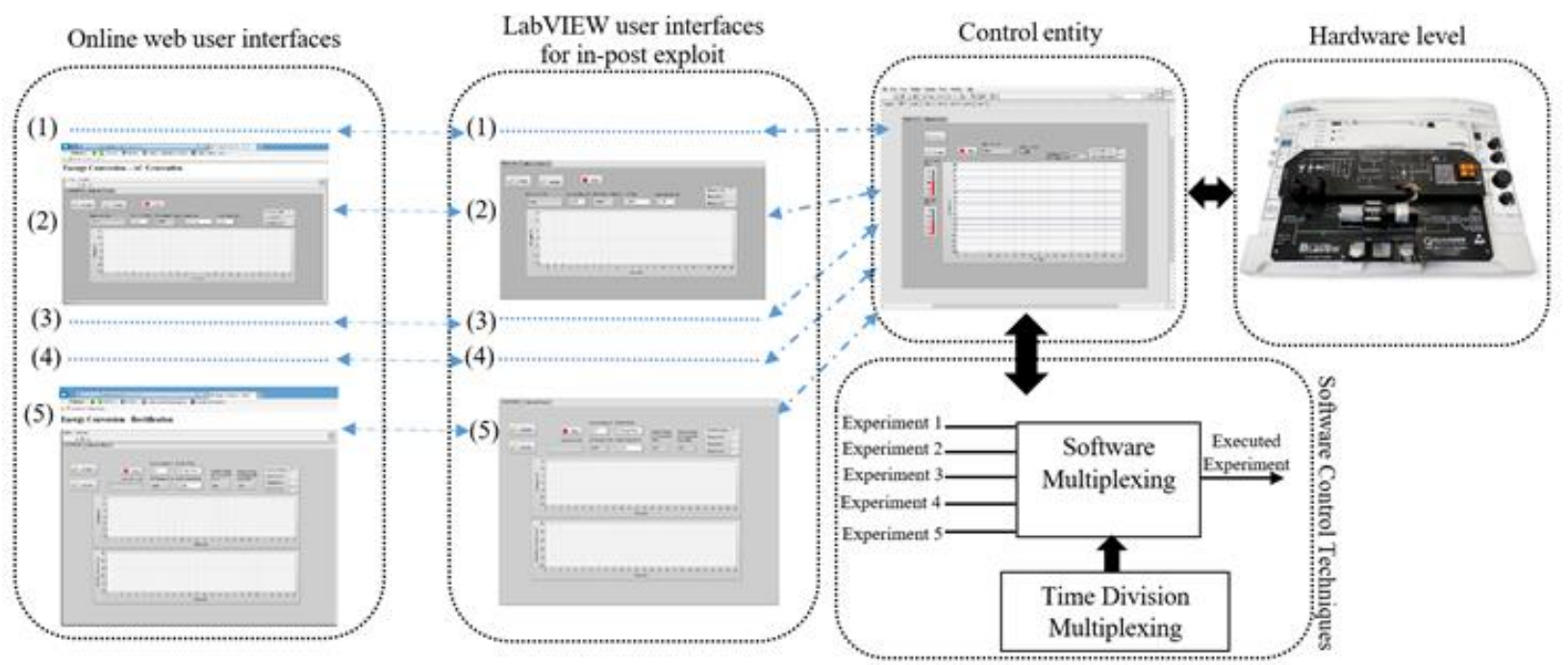

Fig 1:- Developed and adapted structure for remote exploit on NI Elvis and Quanser.

Modifying and adapting already existing materials in hands-on laboratories from local use to online access and remote exploit will offer rich content of resources and wide range of utilities and covered fields of experimenting. However, the resiliency and flexibility of the aimed hardware and software to deploy are the principal factors to define the possibility of their remote exploit, by scaling them from hands-on manipulations and in-post exploit on computers to the online access through the internet.

In this paper, we present the work of adapting handson laboratory's equipment of National Instruments, usually exploited during local experiments in electronic of energy, to be used through the internet for remote exploit and online experimenting. The adaptation was by developing software multiplexing technique and Time Division Multiplexing technique (TDM) in LabVIEW platform (Fig. 1) [10], [11], to switch between the integrated circuits and components within the used hardware, and ensure the simultaneous availability and reliability of its different experiments while using and sharing the same resources (Fig. 1). In addition, we configured and deployed a web server for online access, and we developed different online web user interfaces, to support the online access and remote exploit of hardware resources through the internet.

The developed software multiplexing technique and TDM technique (Fig. 1)[10], [11] enable to share the same hardware and software resources of NI Elvis system and Quanser System between different experiments nearly simultaneously. In addition, those developed techniques enable to share each experiment between different online users, which augment the number of potential experimenters on NI Elvis system and Quanser system.

There are different previous works of adapting specific materials of hands-on laboratories to online experimenting, such as using sensors to conduct specific measurements or using cameras to visualize the collected measurements by oscilloscopes and other instruments [1], [8], [12]. In addition, other previous works tried to use smart devices to control hardware fields of experimenting and adapt them to online exploit [13], [14]. However, the presented work in this paper is the first one that treats the adaptation of NI Elvis system and Quanser system to online use and remote experimenting, and treats the extending of conducted adaptation on larger scales of materials.

This paper treats different aspects of adapting hands-on laboratory's materials to online access and remote experimenting. In addition, it presents the projection of many of those aspects on NI Elvis system and Quanser system (Fig. 1), by adapting them to online access and remote exploit, in order to support higher numbers of experimenters while protecting the hardware resources from fast failure and destruction by minimizing the hands-on contact on them.

This paper is structured as follows: Section 2 presents the requirements to adapt hands-on laboratory experiments to online access and remote exploit. Section 3 presents the work of adapting NI Elvis system and Quanser system to remote experimenting. Section 4 presents our deployed experiments in electronic of energy for online use through the internet. Finlay, section 5 for conclusion and future work.

\section{ADAPTING HANDS-ON LABORATORY EXPERIMENTS TO ONLINE ACCESS AND REMOTE EXPLOIT}

Adapting and deploying hands-on laboratory's hardware for online use and remote experimenting bring many advantages, opportunities and facilities in the fields of scientific research and education. From these varied advantages, there is the remarkable opportunity of sharing hardware and software platforms between different establishments, while increasing the number of students that may experiment on the same resources [15]. In addition, offering the opportunity of using software and hardware multiplexing and switching techniques, to create shared topologies and infrastructures that provide shared online exploit of resources with large combinations of experiments. 
As an example of the attempts of sharing laboratory resources, the elaborated work in [14], where its conductors tried to present and describe an open model of shared laboratories and shared topologies through the internet. There are other relevant works of adapting usual materials of hands-on laboratories to online access by using different approaches of control and monitoring, either by using cameras for visualization or by developing web interfacing utilities or web client modules [1], [8].

Those shared topologies will open the door to different universities and institutions around the world to deploy, use and share their hands-on laboratory's materials through the internet, and collaborate between them in many terms. In addition, they will offer the opportunity to different establishments to have their own web services of online experimenting while exploiting dislocated shared resources, which will reduce the monetary fees of the financial investment aspects of these establishments.

On other hand, there are many technical and conceptual aspects to consider and treat, in order to adapt and deploy any hands-on laboratory's hardware or software for online access, and exploit them in form of remote experiments. From many technical and conceptual questions to search for their answers, there are the next principal interrogating marks on relevant axes:

$>$ Does the aimed hardware for remote access and exploit have interfaces to exchange the data with smart devices and other machines such as computers?

$>$ Does this hardware have its own web interfacing utilities, software frameworks or specific developed applications for its use and control?

$>$ Are those web utilities, software frameworks or applications resilient for changing such as editing, manipulating and modifying to be adaptable to the online use?

$>$ What are the presented physical and software limits, and what are the exploit potentials of the aimed resources of hardware?

$>$ How much the hardware may be considered as flexible and resilient for online access and remote exploit, and what is the supportable number of online access sessions in parallel to be properly managed?

$>$ What is the maximum number of experiment queries that may be handled and served in a simultaneous way?

$>$ Moreover, how much the material resources are reliable, securable and adaptable to online access and remote use while being shared and exploited by multiplicity of remote labs, e-learning web services or other web servers of different establishments?

In addition, there are many other technical and conceptual questions of relevant axes, depending on the perspective of utilizing, the aimed educational or scientific field, and the aimed hardware and software for online access and remote exploit.

\section{A. The Hardware Aspect of Online Experiments}

There are many used materials and instruments in hands-on laboratories dedicated to in-place or in-post experimenting; either exploited through hands-on manipulations or through computers without distance access. They diverse from electrical/electronic components, measurement instruments, power supply systems, workbenches, to a multiplicity of objects and products that depend on the niched fields of experimenting.

Even the non-smart materials and instruments with no support for computer's use, they can be altered and modified. Alternatively, they can be adapted by relying on exterior smart instruments, networking devices or outside sensors to control and manipulate their inputs and transfer their assembled measurements to computers. Otherwise, converting their output results of experiments to other forms of measurable behaviours, events or parameters to be transformed, translated and transferred to computers.

In other hand, there is the solution of using videos or pictures collecting methods by relying on cameras, to visualize the experiment hardware and its execution processes, or visualize the displayed values of used instruments for measurements. This solution may be conveniently reinforced by providing certain ways of online user interfacing, to manage and control the corresponded inputs of variables and parameters.

There are also other elementary and principal technical concepts to consider, such as the use of physical switches to control and manage the power supply. Those switches may be also used to control the interconnections and physical links between the arranged components and materials in binary states $\{0,1\}$, or even switch or multiplex between them for more potential combinations of experiments.

Using this mechanic of hardware switching also opens the way to put the used materials in standby modes. Those standby modes are where the execution of an experiment to apply the modifications of provided parameters, and/or execute the measurements retrieving, will not happen until having certain launching events. Conveniently, those experiment executions should occur only for slights of time depending on the composition and complication of their measurements.

Any of those execution processes of experimenting may be launched only after a conditioned event. This conditioned event may be something like clicking on a one state button on an application. Then, after finishing the experiment execution process, releasing the hardware resources to the benefit of other triggering events of execution to occur and take place will be an essential technical clew to establish the physical supporting ground of multi-access based experiments. In addition, releasing those resources after each execution process will open the way to have multiuser services of remote experimenting, while sharing the exploit of the same hardware and software between users or even between establishments. 
In this context, we are working on developing a global hardware model of adapting the materials of National Instruments (NI) to online access and remote exploit. In addition, we are working on adapting other materials, which are not from National instruments, to be used through the internet for remote exploit. Moreover, we are working on comparing those materials with other resources at the market, to define the most convenient products for remote experimenting. This model is based on modifying and adapting hardware materials dedicated for in-post experimenting on computers, to be used through the internet for educational purposes. This global model is based on extending the presented work in this paper to larger scales (Fig. 1), by adapting it to different resources.

\section{B. The Software Aspect of Online Experiments}

After briefly treating and discussing both of the support and the breakthrough to communicate a prospective experimental hardware with computers, or other potential smart machines and devices, we come to the edge of analyzing the aspect of its logical deployment, and its manner of use and exploit at the software level.

There are many commercial materials, instruments, smart devices and other products in the market provided with their own dedicated software, code sources, applications, web user interfaces or other interfacing utilities to handle their use on laptops or on desktop computers. As an example of these resources: NI (National Instruments) products, Raspberry, Arduino, etc. However, there will be many purchased characteristics and basics in these resources before considering any of them for online access, remote use and multiusers based exploits.

In other hand, hardware markets provide different types and series of processors, smart microchips and microcontrollers, to develop different materials or embedded systems to be used in experimental fields. These resources offer the advantages of having more flexibility at the level of software frameworks, having more adaptability to communicate with various frameworks, and even having the possibility to interact with different programming languages such as $\mathrm{C}++$ and Java.

Therefore, the first interrogation to drop at this point is; are the developed resources at the software level of potential hardware parts editable to be changed and modified to have a remote exploit on them?

The principal goal of this previous interrogation mark is to determine whether the used software resources are proprietary or open resources. In addition, determining how much these software resources may be considered as open and free resources, and what are their involved programming languages?

Those previous interrogations are aiming to search and explore the dimensions of resources resiliency and flexibility to be modified, altered or adapted to the online exploit of the hardware parts.
In this context, concerning the NI Elvis and the Quanser system, which we adapted to online access and remote exploit, they are NI products (National Instruments), and both of them are usually dedicated for in-post exploit on computers. They rely on the use of LabVIEW platform as a software development framework for their use and exploit.

The use of LabVIEW platform (Fig. 1) by NI Elvis system and Quanser system allows having a varied range of modification and manipulation to induct on the manner of utilizing them, since the developed resources of LabVIEW are designed to handle or to be handled by different programming languages; such as $\mathrm{C}++$ programming language and Java.

\section{The Aspect of Online Web Interfacing}

This important part treats the specific concerns of developing web interfacing utilities, or applications, for distance use through the internet. Those interfacing utilities will be required to communicate with specific control entities, or measurement servers, to support the remote executions of experiments through the internet.

Those web interfacing utilities and applications must offer to their users, generally, the hand on manipulating the values of input fields for parameters changing. Then, they must display and visualize the collected results after launching specific conditioned events, such as clicking on a one state button, to execute the modifications of experiments and collect the measurements.

The development process of those web user interfaces, or applications, may generously consider the distributeabillity of the final elaborated products; to be deployed and used through numerous web servers and online web platforms.

One of the principal aims while developing those web interfacing utilities and applications should be to communicate and interact with certain control entities, or measurement servers, in order to exploit the deployed hardware. In addition, it will be significant to develop time multiplexing or time division techniques as logical processes at the software level of remoted experiments; to elevate the supportable number of potential requests of experimenting to be received, managed and properly served without errors or latencies.

There are other essential logical techniques, such as software switching or software multiplexing to control and manage the executed codes of experiments [10]; to switch or multiplex between them in case of using the same components at the hardware level. Those techniques are also recommended to optimize the allocated RAM (Random Access Memory) space and allocated capacities of processing. This optimization will help to handle more incoming requests from different online experiments while relying on the same resources, in addition of reducing the time latencies of experiments serving. 
Developing the web interfacing utilities and web client modules independently from specific web access platforms, with their own configurations to communicate with specific control servers or measurement servers, will be convenient to enable their integration and deployment within various web servers of different establishments. Otherwise, it will be complicated to separate the developed files and functionalities of those interfacing utilities and client modules from their default web infrastructures.

In this context, we used Java to develop the scripts of the utilities of interacting and interfacing between LabVIEW and the developed web user interfaces, while using parallel execution processes of experimenting, to optimize the exploit of processors and RAM resources of the deployed server that hosts those adapted experiments on NI Elvis and Quanser. In addition, we used JavaScript and HTML (Hyper Text Markup Language) to develop the web user interfaces of adapted experiments (Fig. 1).

Those choices of using Java, JavaScript and HTML are due to their simplicity of development and integration within different platforms of software. In addition, they offer the possibility of interacting with different programming languages and frameworks such as LabVIEW.

\section{The Aspect of Online Access Platforms}

Generally, online platforms, or web access platforms, consider the identification and authentication processes, and treat the access aspects of potential users or service visitors. Those processes are necessary to verify and validate the credentials and/or authorizations of service users and visitors, before allowing any of them to access and exploit the provided online services.

Moreover, taking into account the security aspect of transferred data such as login accounts, client passwords and submitted files, is an essential part to provide a secured and integrated online service. This security is essential to stand against the possible attacks of spoofing and sniffing the transmitted data between the client-sides and the databases of access platforms [16]. In addition, it is essential to take into account the use of counter measures and technologies to avoid potential attacks of web intrusions [17], code injection attacks [18], Man In The Middle (MITM) attacks and other types of network intrusions [19].

Concerning the aspect of developing web access platforms for distance experiments, in addition of the previous mentioned points about provided services and security, those access platforms should provide reliable reservation services for scheduling or booking, to manage the experiments time allocation to the online clients and service visitors. In those scheduling or booking services, any potential experiment must be booked or scheduled in order to take place and be served.

In addition, it will be helpful to develop and integrate principal accounts of administrative or instructive access authorities, or other accounts of guest services, to allow having a direct access to the web interfacing utilities or client modules without pre-established reservations. Those accounts will help to teste, or validate, the operability of the provided online experiments before their official exploit by students or by other service clients.

The aspect of online access platforms also opens the door to provide and serve theoretical educational contents for the supported experiments on the deployed hardware, such as using interactive quizzes, videos, documents, PDFs and other types of numerical files to support the pedagogical tasks of distance learning and online training.

In this context, we deployed the Moodle platform [20] to support different services of e-learning and distance experimenting. In addition, we are currently using the Moodle platform to control the scheduling and remote access to the adapted experiments on NI Elvis System and Quanser system.

\section{ADAPTING NI ELVIS SYSTEM AND QUANSER SYSTEM FOR REMOTE EXPERIMENTING}

In this section, we are presenting all conducted works of adapting NI Elvis system and Quanser system to have an online access and remoted exploit on them. In addition, we are presenting and describing all conducted works of developing a software multiplexing technique [10], a Time Division Multiplexing Technique (TDM)[10], [11] and online web user interfaces to exploit NI Elvis system and Quanser system through the internet by multiusers, while sharing the exploit of the same hardware and software.

Relying on the use of software multiplexing techniques to switch and interconnect between different circuits, to combine them in different structures; offers the possibility to use those circuits in different experiments at the software level, while exploiting them through the internet by distant multiusers. However, there is an absolute necessity of having certain hardware switching techniques to switch and interconnect between those circuits, which to be logically managed and executively controlled at the software level. In this context, the Quanser system contains different embedded circuits and consists of integrated physical switchers to control the hardware interconnections between its integrated circuits, to circulate the electrical signal between them in different combinations of experiments.

Using software-multiplexing techniques to interconnect between independent circuits in different structures and combinations opens the way to create different experiments that share the same hardware and software resources. This diversity of structures is an important key to found the pillars of having rich and diverse services of experimenting, in addition of minimizing the quantity of deployed materials. In this context, our developed technique of software multiplexing within LabVIEW platform (Fig. 1) helps to run the codes of all deployed experiments nearly simultaneously while sharing the same resources of hardware and software. In addition, it helps to augment the number of experimenters through the internet while sharing the same resources between them. 
The development process of a software multiplexing technique is absolutely dependent on using a time division multiplexing technique, or the development of specific time division and control methods; to enable the exploit of the hardware parts by different users simultaneously or nearly simultaneously [10], [11]. On other hand, there is an absolute necessity to liberate the hardware resources after each executed event of experimenting and measurements conducting, to open the way to exploit the hardware parts and utilize the shared software resources by other users.

We developed a software multiplexing technique within LabVIEW platform (Fig. 1), where we developed interpolation code scripts using the concept of Threads in Java to launch each experiment independently and interact with the web user interfaces in parallel modes of execution. In addition, we developed a technique of Time Division Multiplexing (TDM) within LabVIEW platform (Fig. 1), where time is devised between experiments to allocate specific time slots of microseconds to each experiment to exploit and share the hardware resources. Moreover, we used software loops (While loops) within LabVIEW platform, to run all the experiments continuously in standby modes where each experiment is waiting the execution orders from online users for parameters modification and measurements execution.

Each developed code of adapted experiments is characterized by having different time slots of microseconds for each process of experimenting. The maximum number of supported experimenters on each experiment is defined to ten experimenters, to deploy a stable service of remote experimenting on NI Elvis and Quanser (Fig. 2).

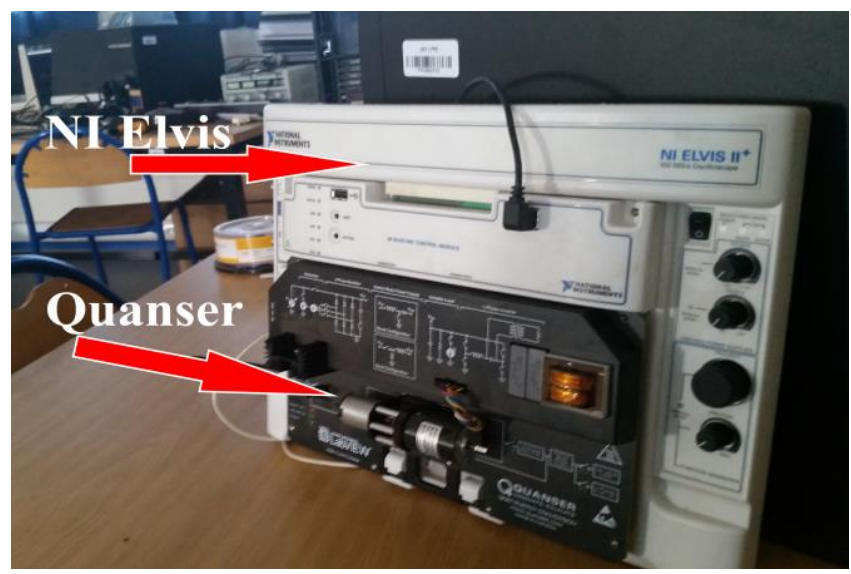

Fig. 2:- The NI Elvis System and Quanser system.

\section{Experiments time responses in function of the number of online experimenters}

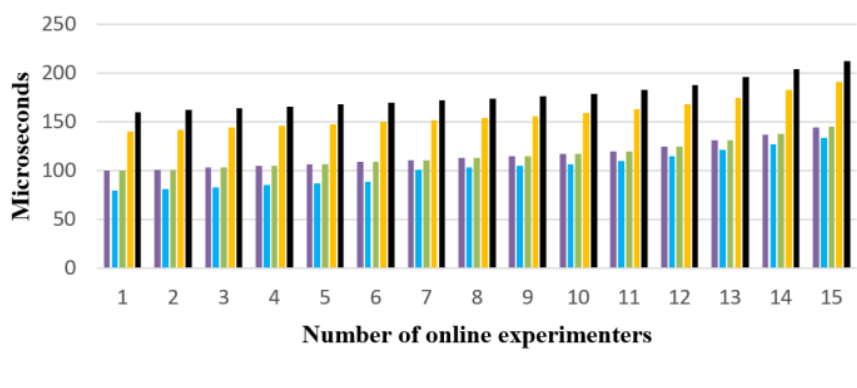

aExperiment 1 axperiment 2 Experiment 3 - Experiment 4 axperiment 5 Experiment 1: experiment of 3-phases power generation.

Experiment 2: experiment of AC (Alternating Current) generation. Experiment 3: boost converter experiment.

Experiment 4: signal inverting experiment.

Experiment 5: signal rectifying experiment.

Fig. 3:- Experiments time responses in function of the number of online experimenters on NI Elvis and Quanser.

The maximum numbers of supported experimenters on NI Elvis and Quanser (Fig. 2) were determined after conducting different tests on the deployed experiments, while using Wireshark to calculate the average time responses in function of the numbers of online experimenters (Fig. 3), to make sure that each execution process of experimenting is stable and fast.

During those conducted tests, we relied on varying the numbers of online experimenters on each experiment (Fig. 3 ); to define the stable points were each experiment hade stable comportments and fast executions while being shared between multiusers.

Each developed process of parameters modification on adapted experiments is allocated with a time slot of ten microseconds to be executed. Each developed process of voltage measuring is characterized with a time slot of ten microseconds to be executed. Each developed process of current measuring is characterized with a time slot of ten microseconds to be executed. Finally, each developed process of signal wave collecting of voltage or current is characterized with a time slot of twenty microseconds to be executed.

The already presented choices of time slot intervals was defined after conducting many tests while using Wireshark, to define the optimal choices of time slots for each process. Those choices of time slots were the best used values of time intervals during the conducted processes of online experiments testing while using Wireshark, because they enabled us to have the presented results in Fig. 3, which were the best collected time responses of experimenting while we were augmenting the numbers of online experimenters on NI Elvis and Quanser. 
Before our conducted optimizations by developing the software multiplexing technique and the Time Division Multiplexing technique within LabVIEW platform (Fig. 1), our deployed NI Elvis system and Quanser system (Fig. 2) were only computer based-use systems for in-post exploit. They were dedicated for hands-on laboratories where the limit of only using one experiment at a time, with no possibility of smoothly passing from one experiment to others, nor exploiting the same hardware resources in different experiments at the same time ranges and in parallel.

Before our conducted optimizations, the previously mentioned limitations of NI Elvis and Quanser did complicate the possibility of the online use of their resources by different experimenters nearly simultaneously. Therefore, we developed the software multiplexing technique and TDM technique within LabVIEW framework (Fig. 1), to enable the use of the hardware parts and software entities in different structures and combinations nearly simultaneously, which help us to increase the numbers of potential experimenters on NI Elvis and Quanser.

The resources sharing of NI Elvis and Quanser is based on the developed Time Division Multiplexing technique within LabVIEW platform, where each experiment is allocated with specific time slots of microseconds to run its code scripts and exploit the software and hardware resources of NI Elvis system and Quanser system. Then, at the finish of allocated time slots of each executed experiment, the hardware and software resources are liberated to be exploited by other experiment executions.

The developed Time Division Multiplexing Technique (TDM) is to exploit the hardware and software resources by each experiment at specific time slots using while loops conditioned by time intervals. At the finish of allocated time slots of each experiment, the developed technique of TDM liberate all the resources of NI Elvis and Quanser to be exploited by other experiments.

We developed independent variables for each parameter to control and for each measurement to conduct during the supported online experiments on NI Elvis system and Quanser system (voltage, current, signal wave, signal frequency, resistance values, capacitor values, etc.). Those variables are shared between deployed experiments, because they exploit the same hardware resources and software resources of NI Elvis and Quanser. Thereby, those experiments have the same measurements of voltage, current and signal waves to collect, but they have different combinations of circuits to experiment while sharing the same resources of hardware and software.

We developed independent user interfaces in LabVIEW platform (Virtual Interfaces) as shown in Fig. 1, to control the parameters and retrieve the measurements of experiments in resilient and flexible ways while using the already described techniques of software multiplexing and Time Division Multiplexing.
We developed the online web user interfaces using HTML (Hyper Text Markup Language) and JavaScript (Fig. 1), for the remote experimenting on NI Elvis system and Quanser system. In addition, we developed the interfacing utilities between LabVIEW and the web user interfaces using Java, to manage the values communication of parameters and measurements between LabVIEW and online users in parallel modes of execution.

The developed interfacing utilities between LabVIEW and web user interfaces are characterized by using a Class script in Java for each parameter or variable to control, and executing each script as a Thread process to run at the background of the experiments hosting server in parallel modes.

We deployed and configured a web server, to host the developed web interfaces and support the online access and remote exploit of the hardware resources of NI Elvis system and Quanser system through the internet.

As a result, we created the possibility of the online exploit of different experiments in electronic of energy, while relying on using and sharing the same hardware and software resources between different experiments in parallel modes of execution.

The resulted developed topology of adapted experiments is as described in Fig. 1. The levels of this topology of remote exploit and online experimenting are as follow:

> The Ni Elvis and the Quanser system at the hardware level with their integrated hardware switches and integrated circuits, to be exploited locally on computers using the local network of our remote lab, and to be exploited through the internet.

$>$ The developed control entity at the software level, which integrates the developed techniques of TDM and software multiplexing (Fig. 1) [10], [11]. This control entity is developed to control and multiplex between the hardware and software parts of NI Elvis system and Quanser system, to share them between all experiments. In addition, it is developed to be responsible of controlling the parameters of circuits and collecting the results of measurements.

$>$ The developed user interfaces in LabVIEW platform as independent entities (Virtual Interfaces), where each experiment has its own user interface for local in-post use in our hands-on laboratory.

$>$ Finally, the developed web user interfaces in JavaScript and HTML for online use and remote experimenting while using Java to communicate them with LabVIEW; enriching both of our in-post experimenting content and our supported online service of remote experiments.

We are working on developing a global model of adapting hands-on laboratory's materials of National Instruments to online access and remote experimenting, by extending the presented work in this paper. In addition, adapting other resources that are not from National Instruments by using sensors, Arduino and microcontrollers. 
In this global model, we are working on developing an equipment server in LabVIEW framework, resembling to the control entity in Fig. 1, which to be adaptable to different instruments and materials of NI, and other instruments such as Arduino and Microcontrollers. This equipment server is aimed to control and monitor the hardware field, apply the changes of variables and input parameters, and collect the results of measurements.

In addition, we are working on developing a measurement server responsible of receiving the requests of experimenting from online users, and then forwarding those requests to the equipment server. This measurement server is based on the already presented work in this paper about using Java to communicate the web user interfaces (Fig. 1) with the developed control entity in LabVIEW.

After the measurements conduction during any experiment, the equipment server will be responsible of forwarding the collected measurements to the measurement server, which will be responsible of sending collected results to experimenters.

The global model that we are working on to adapt the already mentioned materials of hands-on laboratories to remote experimenting is inspired from the used approach on VISIR system (Virtual Instrument Systems In Reality) [21]. The inspired approach from VISIR relies on using an equipment server to control and monitor the hardware field, and relies on a measurement server to manage the online requests of experimenting.

There is a considerable number of other optional solutions to elaborate and develop the control interfaces of experiments (Fig. 1), the equipment server side and the measurement server side; such as relying on the use of Matlab framework [22], Simulink [23], Scicos [24], C++ programming language [25], etc. However, latency, service yield, efficiency, the number of supportable simultaneous users and online security will be always the main characterizing factors of any developed entity for online access and remote experimenting.

\section{DEPLOYED EXPERIMENTS IN ELECTRONIC OF ENERGY FOR REMOTE EXPLOIT}

After developing the software multiplexing technique, the TDM technique, the LabVIEW user interfaces, the online experiment server and the web user interfaces, we deployed the resulted adapted experiments for online use. As a result, there are five principal experiments in electronic of energy carried out through our remote lab's service for local in-poste use in our remote lab and for remote experimenting through the internet by multiusers.

Each deployed experiment has different time of execution according to the number of measurements to conduct. Therefore, after numerous tests and modifications on the allocated time slots of execution, we are currently supporting 10 online experimenters on each deployed experiment as a maximum.
We configured the number of supportable users on each experiment to be 10 experimenters as a maximum, because we need to conduct further processes of optimizing and testing before augmenting those numbers of supportable experimenters.

We used the Wireshark to calculate the average time of execution of each deployed experiment. Those calculations were collected during the conduction of numerous tests on the deployed systems of NI Elvis and Quanser, where the conduction of each experiment from various numbers of online experimenters (Fig. 3).

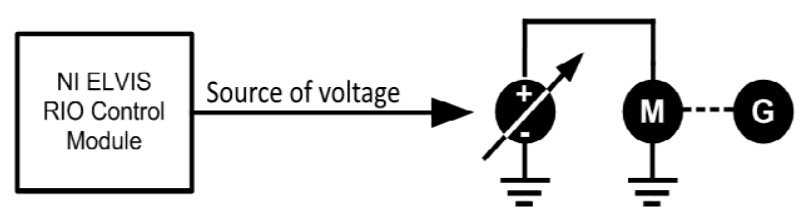

Fig. 4:- Experiment Circuit schema of 3-phases generator experiment.

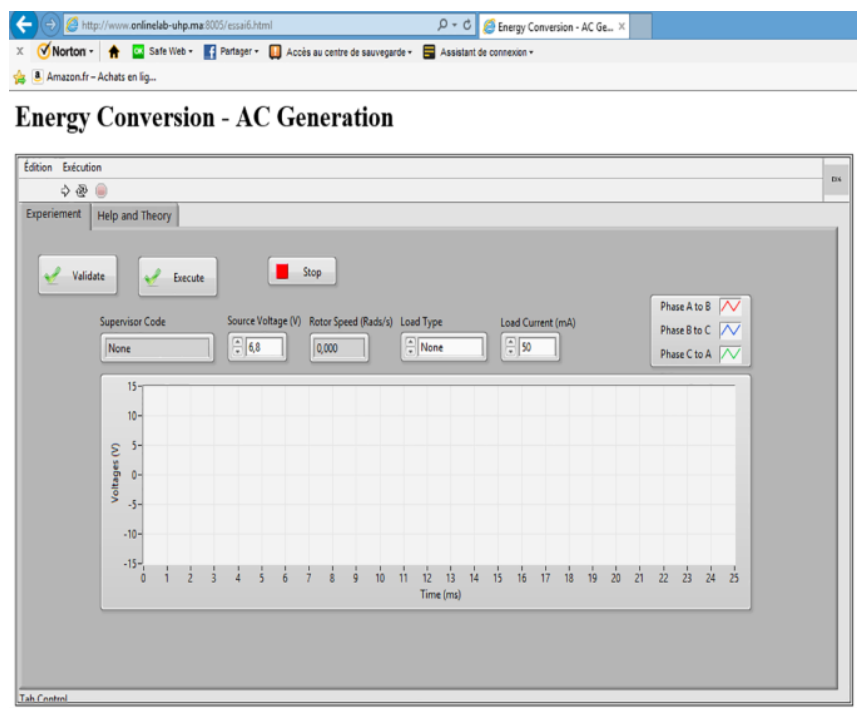

Fig. 5:- Web user interface for the experiment of 3-phases generating.

The developed Experiment 1 on NI Elvis and Quanser (Fig. 3), which is an online experiment of 3-Phases power generating, relies on an integrated generator within the Quanser system (Fig. 4), to convert one single phase of electrical signal to 3-phases of signal. The developed web user interface for this experiment is as shown in Fig. 5. This user interface gives the hand on manipulating the source voltage (Fig. 4), the load type (resistance or none) and the load current value. On other hand, it displays the rotor speed of the integrated generator and the output voltage values. The calculated average time of execution of this experiment by using Wireshark is 100 microseconds. The configured maximum number of experimenters on this experiment (Experiment 1) is 10 users. Those 10 users are including online users and local in-post users. 
The developed Experiment 2 on NI Elvis and Quanser (Fig. 3), which is an online experiment of AC (Alternating Current) transformer, relies on an integrated transformer within the Quanser system to be exploited through the developed web user interface shown in Fig. 6. This experiment aims to manipulate and convert the energy between the supplied input power by Ni Elvis and the output of the transformer, while supplying a flexible load at the output as shown in Fig. 7. The calculated average time of execution of this experiment by using Wireshark is 80 microseconds. The configured maximum number of experimenters on this experiment (Experiment 2) is 10 users. Those 10 users are including online users and local inpost users.

The developed Experiment 3 on NI Elvis and Quanser (Fig. 3), which is an online experiment of Boost converter, relies on an integrated boost converter circuit within the Quanser system, to step up the voltage value while stepping down the current value of the power supply. The calculated average time of execution of this experiment by using Wireshark is 100 microseconds. The configured maximum number of experimenters on this experiment (Experiment 3 ) is 10 users. Those 10 users are including online users and local in-post users.

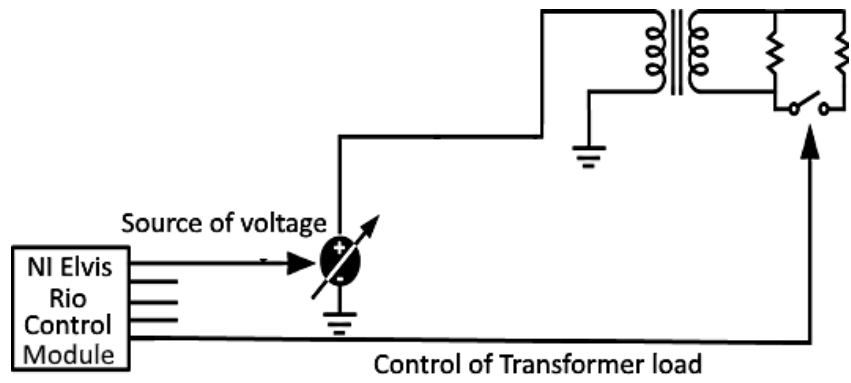

Fig. 6:- Experiment Circuit schema of AC transformer experiment

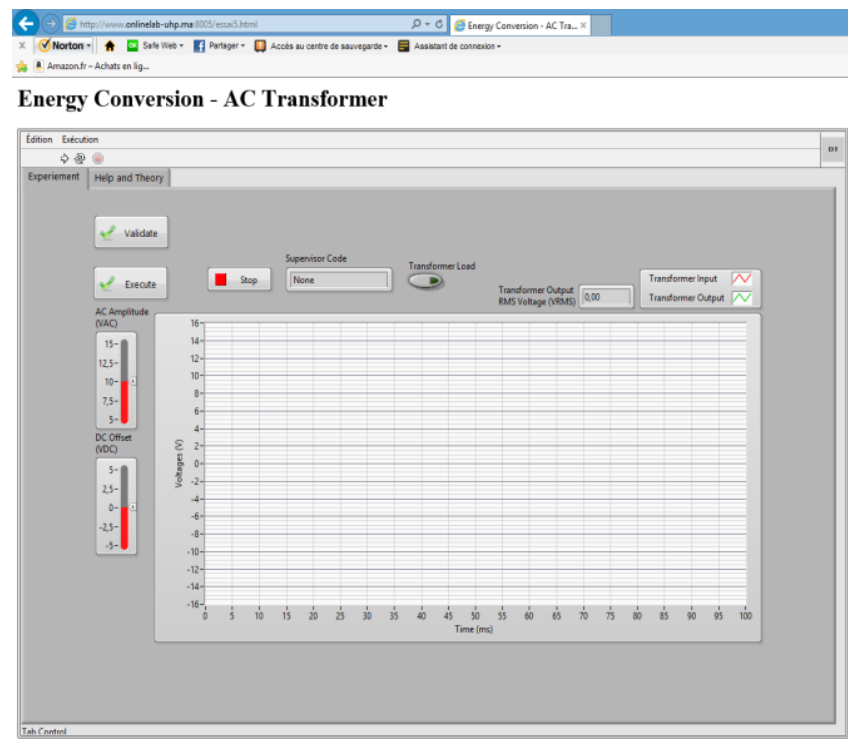

Fig. 7:- Web user interface for the experiment of AC transformer.
The developed Experiment 4 on NI Elvis and Quanser (Fig. 3), which is an online experiment of signal inverting, relies on an inverter circuit within the Quanser system. This circuit is relied on to convert the DC (Direct Current) power source of NI Elvis into a periodic AC (Alternating Current) power supply at the output of the inverter. The calculated average time of execution of this experiment by using Wireshark is 140 microseconds. The configured maximum number of experimenters on this experiment (Experiment 4) is 10 users. Those 10 users are including online users and local in-post users.

The developed Experiment 5 on NI Elvis and Quanser (Fig. 3), which is an online experiment of signal rectifying, relies on a rectifier circuit within the Quanser (Fig. 8), to convert the current of AC power supply to a current of DC supply. The developed web user interface for this experiment is as shown in Fig. 9. The calculated average time of execution of this experiment by using Wireshark is 160 microseconds. The configured maximum number of experimenters on this experiment (Experiment 5) is 10 users. Those 10 users are including online users and local inpost users.

The developed shared exploit on NI Elvis and Quanser is based on resources sharing between experiments, in addition of sharing each experiment between multiusers through the internet. This sharing is relying on the developed techniques of Software multiplexing and Time Division Multiplexing, while using the developed web user interfaces through the internet.

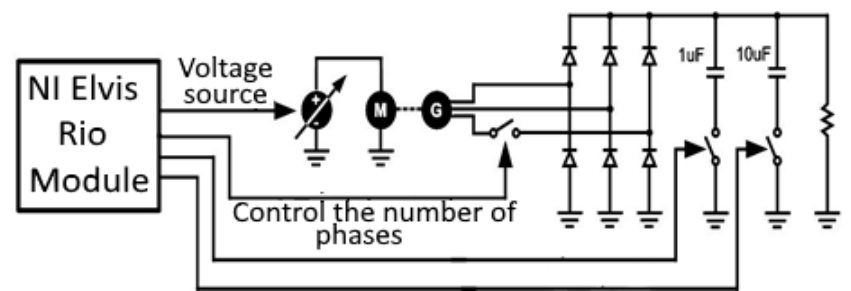

Fig. 8:- Circuit schema of the rectifier experiment.

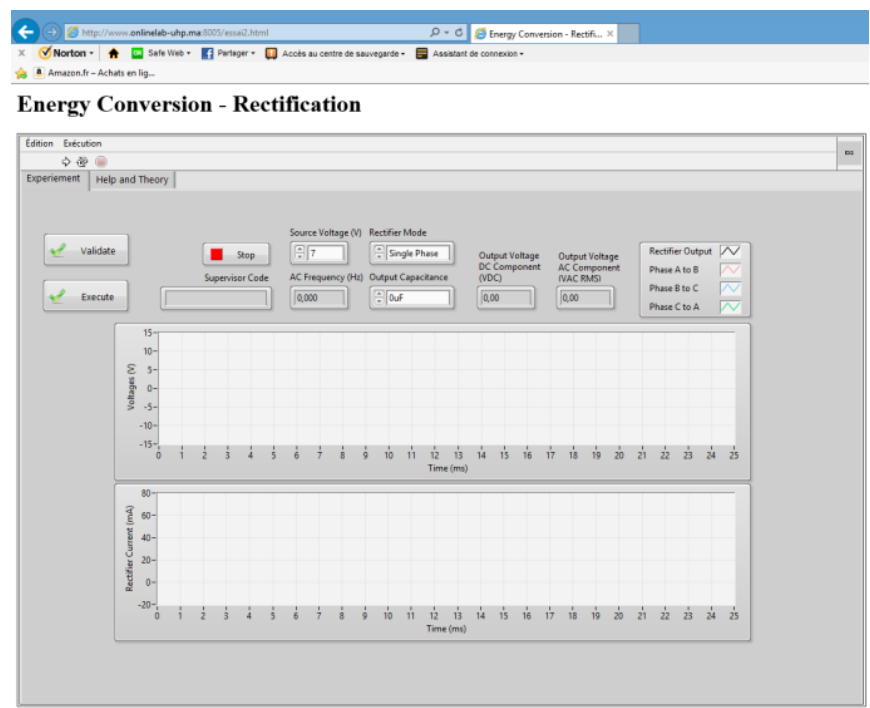

Fig. 9:- Online web user interface for the rectifier experiment. 
The developed techniques of Software multiplexing and Time Division Multiplexing (TDM) help us to augment the number of online experimenters on each deployed experiment. Currently, each developed experiment on NI Elvis and Quanser is configured to have ten online experimenters as the maximum number of supportable experimenters, to provide stable and fast services of experimenting. However, we need to conduct additional optimizations and tests to be able to augment the numbers of experimenters higher than the current reached levels.

\section{CONCLUSION}

The conducted work on our hands-on laboratory's materials (NI Elvis and Quanser) to adapt them to the online use and remote exploit; enables us to support high numbers of experiments from different distant users at the same time. In addition, it prevents the excess of hands-on contact and manipulation on those materials, which delays their destruction and failure. Therefore, projecting the presented work in this paper on larger scales of materials is significant, to support higher numbers of experimenters while sharing the same resources between them, in addition of protecting the hardware resources from excess of handson contact.

\section{REFERENCES}

[1]. J. Ma, J. V. Nickerson, "Hands-on, simulated, and remote laboratories: A comparative literature review," J. ACM Computer Surveys, vol. 38, pp. 1-24, 2010.

[2]. Y. H. Elawady, A. S. Tolba, "Educational objectives of different laboratory types: A comparative study," Int. J. Comput. Sci. Inform. and Security, vol. 06, pp. 89-96, 2009.

[3]. N. S. Edward, "The role of laboratory work in engineering education: Student and staff perceptions," Int. J. Electrical Eng. Educ, vol. 39, issue 1, pp. 1119, 2002.

[4]. J. Brüggemann, K. Bizer, "Laboratory experiments in innovation research: a methodological overview and a review of the current literature," Journal of Innovation and Entrepreneurship, vol. 5, issue 24, pp. 1-13, 2016.

[5]. G. G. Karady, "Roll of laboratory education in electrical power engineering education," presented at IEEE Power and Energy Society General Meeting Conversion and Delivery of Electrical Energy in the $21^{\text {st }}$ Century, Pittsburgh, PA, USA, IEEE, 2008.

[6]. Y. Zhu, J. B. Tracy, J. Dong, M. G. J. X. Jiang, G. Childers, "Teaching a multidisciplinary nanotechnology laboratory course to undergraduate students," Journal of Nano Education, vol. 5, pp. 1726, 2013

[7]. P. Orduña, D. G. Zutin, S. Govaerts, I. L. Zorrozua, P. H. Bailey, E. San, “An extensible architecture for the integration of remote and virtual laboratories in public learning tools," IEEE Revista Iberoamericana de Tecnologias del Aprendizaje, vol. 10, pp. 223-233, 2015.
[8]. S. A. Shanab, S. Odeh, R. Hodrob, M.Anabtawi, "Augmented reality internet labs versus hands-on and virtual labs: A comparative study," Proc. Int. Conf. Interactive Mobile and Computer Aided Learning, Amman, Jordan, IEEE, pp. 17-21, 2012.

[9]. M. Tawfik, D. Lowe, C. Salzmann, D. Gillet, E. Sancristobal, M. Castro, "Defining the critical factors in the architectural design of remote laboratories," IEEE Revista Iberoamericana de Tecnologias del Aprendizaje, vol. 10, pp. 269-279, 2015.

[10]. A. Minaeva, P. `S`ucha, B. Akesson, Z. Hanza'lek, "Scalable and efficient configuration of time division multiplexed resources," Journal of Systems and Software, vol. 113, pp. 44-58, 2016.

[11]. S. Faruque, "Time division multiplexing (tdm)," in: Radio Frequency Source Coding Made Easy, Springer, pp. 91-118, 2015.

[12]. M. Stefanovic, V. Cvijetkovic, M. Matijevic, V. Simic, "A labview-based remote laboratory experiments for control engineering education," Comput. Appl. Eng. Educ, vol. 19, pp. 538-549, 2011.

[13]. J. Chac'on, H. Vargas, G. Farias, J. S'anchez, S. Dormido, Ejs, jil server, and labview: An architecture for rapid development of remote labs, IEEE Trans. Learn. Technol, vol. 8, pp. 393-401, 2015.

[14]. M. Chirico, A. M. Scapolla, A. Bagnasco, "A new and open model to share laboratories on the internet," IEEE Trans. Instrum. Meas, vol. 54, pp. 1111-1117, 2005.

[15]. P. Orduña, J. García-Zubia, D. López-de-Ipiña, P. H. Bailey, J. L. Hardison, K. DeLong, V. J. Harward, "Sharing laboratories across different remote laboratory systems," IEEE 12th International Conference on Advanced Learning Technologies, Rome, Italy, IEEE, 2012.

[16]. Y. Chen, "Security risks and protection in online learning: A survey," International Review of Research in Open and Distance Learning, vol. 14, pp. 108-127, 2013.

[17]. V. R. Mouli, K. P. Jevitha, "Web services attacks and security- a systematic literature review," Procedia. Comput. Sci,, vol. 93, pp. 870-877, 2016.

[18]. T. K. Georgea, K. P. Jacob, R. K. James, “Token based detection and neural network based reconstruction framework against code injection vulnerabilities," J. Inform. Secur. Appl, vol. 41, pp. 75-91, 2018.

[19]. C. Kiennert, N. D. Vos, M. Knockaert, J. GarciaAlfaro, "The influence of conception paradigms on data protection in e-learning platforms: A case study," IEEE Access, vol. 7, pp. 64110-64119, 2019.

[20]. G. C.Oproiu, "A study about using e-learning platform (moodle) in university teaching process," Procedia. Soc. Behav. Sci,, vol. 180, pp. 426-432, 2015.

[21]. J. G. Zubia, J. Cuadros, S. Romero, U. H. Jayo, P. Orduña, M. Guenaga, L. G. Sabate, I. Gustavsson, "Empirical analysis of the use of the visir remote lab in teaching analog electronics," IEEE Trans. Educ, vol. 60, pp. 149-156, 2017. 
[22]. G. Farias, R. D. Keyser, S. Dormido, F. Esquembre, "Developing networked control labs: A matlab and easy java simulations approach," IEEE Trans. Ind. Electron, vol. 57, pp. 3266-3275, 2010.

[23]. E. Fabregas, G. Farias, S. Dormido-Canto, S. Dormido, F. Esquembre, "Developing a remote laboratory for engineering education," Comput. \& Educ, vol. 57, pp. 1686-1697, 2011.

[24]. Z. Magyar, K. Zakova, "Scilab based remote control of experiments," IFAC Proceedings Volumes, vol. 45, pp. 206-211, 2012.

[25]. R. C. Castello', M. Valles, L. M. Jimenez, L. DiazGuerra, A. Valera, R. Puerto, "Integracion de dispositivos fisicos de un laboratorio remoto de control mediante diferentes plataformas: Labview, matlab y c/c++," Revista Iberoamericana de Automtica e Informtica Ind. (RIAI), vol. 07, pp. 2334, 2010. 